\title{
THE SUMS OF POWERS THEOREM FOR COMMUTING BLOCK MAPS
}

\author{
BY
}

FRANK RHODES

\begin{abstract}
A block map is a map $f:\{0,1\}^{n} \rightarrow\{0,1\}$ for some $n \geqslant 1$. A block map $f$ induces an endomorphism $f_{\infty}$ of the full 2-shift $(X, \sigma)$. Composition of block maps is defined in such a way that $(f \circ g)_{\infty}=f_{\infty} \circ g_{\infty}$. In this paper some recent results concerning the set $\{g \mid g \circ f=f \circ g\}$ for certain types of block maps $f$ are extended.
\end{abstract}

It has been shown [2, Theorem 3.4] that the endomorphisms of the shift dynamical system over $\{0,1\}$ can be studied via block maps. An $n$-block is a sequence $b_{1} \ldots b_{n}$ where $b_{i} \in\{0,1\}$ for $1 \leqslant i \leqslant n$, and an $n$-block map is a function from the set of $n$-blocks to the set $\{0,1\}$. Two endomorphisms commute if and only if the corresponding block maps commute. There is a 1-1 relationship between $n$-block maps and polynomial functions in $n$ variables over $Z_{2}$ [2, Theorem 19.1]. The commuting block maps problem has been solved in [1] for linear block maps and for block maps with polynomials of the form

$$
f\left(x_{0}, \ldots, x_{k}\right)=x_{0}+\prod_{i=1}^{k}\left(x_{i}+\delta_{i}\right)
$$

$k \geqslant 2$, where $\delta_{1} \ldots \delta_{k}$ is a $k$-block which is independent of the variables. It is shown that if the least period of $\delta_{1} \ldots \delta_{k}$ is no greater than $\frac{1}{2} k$ then the block maps which commute with $f$ are the powers of $f$, while if the least period of $\delta_{1} \ldots \delta_{k}$ exceeds $\frac{1}{2} k$ then a block map commutes with $f$ if and only if it is the sum of an odd number of powers of $f$. The first step in the proof of these results is to establish the Sums of Powers Theorem which states that if $g$ commutes with $f$ then it is the sum of an odd number of powers of $f$. The Sums of Powers Theorem is proved in [1] for block maps $f\left(x_{0}, \ldots, x_{k}\right)=x_{0}+\phi\left(x_{1}, \ldots, x_{k}\right), k \geqslant 2$, for which the full product $x_{1} \ldots x_{k}$ is a summand of $\phi\left(x_{1}, \ldots, x_{k}\right)$.

It is clear that the Sums of Powers Theorem cannot hold for a block map $f$ which is itself a power of some other block map. In this paper I isolate a property of block maps which are linear in the first variable and prove that for each such block map $f$ no power $f^{n}, n \geqslant 2$, has that property, while if $f$ does have that property then a form of the Sums of Power Theorem holds for $f$. As a corollary, the complete solution of

Received by the editors October 23, 1979 and, in revised form, February 23, 1981.

1980 Mathematics Subject Classification. Primary 54H20. 
the commuting block maps problem obtained in [1] is generalized to block maps with polynomials of the form

$$
f\left(x_{0}, \ldots, x_{m k}\right)=x_{0}+\prod_{i=1}^{k}\left(x_{m i}+\delta_{i}\right), \quad k \geqslant 2, m \geqslant 1 .
$$

The formal definition of the property will be given later in terms of operators on block maps of the type introduced in [1]. Here the property will be illustrated in the more familiar polynomial notation. Given a polynomial which is linear in the first variable one can write it in a form which shows the initial linear terms, the first nonlinear variable with its quotient, and the remainder. For example,

$$
x_{0}+x_{3}+x_{4}+x_{5}\left(x_{7}+x_{12}+x_{9} x_{10}+x_{9} x_{11}+x_{9} x_{10} x_{13}\right)+x_{6}+x_{7} x_{8}+x_{14}
$$

has $x_{5}$ as its first nonlinear variable with quotient $x_{7}+x_{12}+x_{9} x_{10}+x_{9} x_{11}+$ $x_{9} x_{10} x_{13}$ and remainder $x_{6}+x_{7} x_{8}+x_{14}$. This quotient can be written as

$$
x_{7}+x_{9}\left(x_{10}+x_{11}+x_{10} x_{13}\right)+x_{12}
$$

to show its first nonlinear variable $x_{9}$ with quotient $x_{10}+x_{11}+x_{10} x_{13}$ and remainder $x_{12}$. Again this quotient can be written as

$$
x_{10}\left(1+x_{13}\right)+x_{11}
$$

to show its first nonlinear variable $x_{10}$ with quotient $1+x_{13}$ and remainder $x_{11}$. In this case $x_{5} x_{9} x_{10}$ is a product with the following properties. It starts with the first nonlinear variable of the function. Each subsequent variable is the first nonlinear variable in the quotient of the product of the previous variables. Finally, the quotient of the whole product is linear. Such a sequence $x_{s_{1}} x_{s_{2}} \ldots x_{s_{n}}$ is defined uniquely for each function. The property used in this paper is that $s_{i}-s_{i-1} \leqslant s_{1}$ for all $i$ such that $2 \leqslant i \leqslant n$. In the example, $9-5 \leqslant 5$ and $10-9 \leqslant 5$. We conclude from the theorems to be proved that this block map is not a power of any other block map and that each block map which commutes with it differs by at most a constant from a sum of an odd number of its powers.

1. Preliminaries. The results of [1] will be referred to by their two place reference numbers. Some small changes in terminology will be noted later. The notation of [1] will be used throughout. For the convenience of readers some of the definitions and results of that paper will be repeated here. Those marked with an asterisk are simple generalizations of the results presented in [1]. On many occasions in this paper there will occur pairs of block maps which differ only by a constant. To ease the presentation of some of the results and proofs I introduce the notation $f \simeq g$ to mean $f+g \in Z_{2}$.

The set $\mathscr{F}_{n}$ is the set of all $n$-block maps, i.e., maps from the set of sequences of length $n$ (with entries in $Z_{2}$ ) to $Z_{2}$. The map $I \in \mathscr{F}_{1}$ is defined by $I\left(x_{1}\right)=x_{1}$. If $f \in \mathscr{F}_{m}, g \in \mathscr{F}_{n}$ then $f+g \in \mathscr{F}_{N}, f \cdot g \in \mathscr{F}_{N}$ where $N=\max (m, n)$, and $f \circ g \in \mathscr{F}_{m+n-1}$. We have

$$
(g+h) \circ f=(g \circ f)+(h \circ f), \quad(g \cdot h) \circ f=(g \circ f) \cdot(h \circ f) .
$$

If $f \in \mathscr{F}_{n}$ then $T f \in \mathscr{F}_{n+1}, \quad Q f \in \mathscr{F}_{n-1}, \quad R f \in \mathscr{F}_{n-1}$ are defined by $T f(0 B)=T f(1 B)=f(B), Q f(B)=f(0 B)+f(1 B), R f(B)=f(0 B)$. The left extent 
of $f$ is $\lambda(f)=\max \left\{n \mid f=T^{n-1} g\right.$ for some $\left.g\right\}$. The right extent of $f$ is $\rho(f)=\min \left\{n \mid f \in \mathscr{F}_{n}\right\}$. A block map such that $\lambda(f)=\rho(f)$ is said to be trivial.

[1] (1.1) (a) $T(f+g)=T f+T g$.

(b) $T(f \cdot g)=T f \cdot T g$.

(e) $T(g \circ f)=T g \circ f=g \circ T f$.

(f) $f=g$ if and only if $T f=T g$.

[1] (1.3) Suppose $f$ is not constant and $f \in \mathscr{F}_{n}$. Then

(a) $\lambda(T f)=\lambda(f)+1, \rho(T f)=\rho(f)+1$.

(b) $1 \leqslant \lambda(f) \leqslant \rho(f) \leqslant n$.

(c) $f=T^{\lambda(f)-1} h$ for some $h$ with $\lambda(h)=1$.

(e) $\rho(f+g) \leqslant \max \{\rho(f), \rho(g)\}$. Equality holds if $\rho(f) \neq \rho(g)$.

[1] (2.1) (a) $Q(f+g)=Q f+Q g$. $R(f+g)=R f+R g$.

(b) $Q T=0$.

(c) $R T f=f$ for all $f$.

[1] (2.2) If $f$ is not constant, then $\rho(Q f) \leqslant \rho(f)-1$.

[1] (2.3) $f=I \cdot T Q f+T R f$ and the expression is unique.

[1] (2.9)* If $Q g \in Z_{2}$ then $Q(f \circ g)=Q g \cdot(Q f \circ g)$.

The set $L_{1}$ is the set of block maps $f$ such that $Q f=1$, i.e., $f=I+T R f$.

If $f \in \mathcal{L}_{1}$ then $R(g \circ f)=R f \cdot(Q g \circ f)+R g \circ f$ (see proof of [1] (2.9)).

[1] (2.10) If $f \in \mathcal{L}_{1}$ and $g$ is not constant then $\rho(g \circ f)=\rho(g)+\rho(f)-1$.

[1] (2.11)* If $f \in \mathcal{L}_{1}$ is nontrivial and $g \circ f \simeq g$, then $g$ is constant.

The quotient and remainder operators $Q$ and $R$ will be generalized as follows.

If $f \in \mathscr{F}_{n}$ define $Q_{i} f \in \mathscr{F}_{n-i}$ and $R_{i} f \in \mathscr{F}_{n-i}, 1 \leqslant i \leqslant n$, by

$$
Q_{i} f\left(x_{i+1}, \ldots, x_{n}\right)=f\left(0^{i-1}, 0, x_{i+1}, \ldots, x_{n}\right)+f\left(0^{i-1}, 1, x_{i+1}, \ldots, x_{n}\right)
$$

and

$$
R_{i} f\left(x_{i+1}, \ldots, x_{n}\right)=f\left(0^{i}, x_{i+1}, \ldots, x_{n}\right) .
$$

Here $0^{i}$ stands for a block of 0 's of length $i$.

The properties of $Q_{i}$ and $R_{i}$ listed below follow easily from the definitions.

(1.1) Lemma. (a) $Q_{1}=Q, R_{1}=R$ and $R_{i}=R^{i}$.

(b) $Q_{i}$ and $R_{i}$ are additive, i.e., $Q_{i}(f+g)=Q_{i} f+Q_{i} g$ and $R_{i}(f+g)=R_{i} f+R_{i} g$.

(c) $Q_{s} R_{t} f=Q_{s+t} f$.

(d) If $i-k>0$ and $j-k>0$ then $Q_{i} T^{j} f=Q_{i-k} T^{j-k} f$.

(e)

$$
Q_{i} T^{j} f= \begin{cases}Q_{i-j} f, & j<i \\ 0, & j \geqslant i\end{cases}
$$

A block map $\phi$ will be said to be linear in the first $s-1$ variables if and only if $Q_{i} \phi \in Z_{2}$ whenever $1 \leqslant i \leqslant s-1$. The condition can be written also as $\rho\left(Q_{i} \phi\right)=0$ whenever $1 \leqslant i \leqslant s-1$. Note that some modification of the terminology of [1] is involved here. In this paper $f$ will be said to be linear in the first variable if 
$Q_{1} \phi \in Z_{2}$. Nevertheless, the notation $\mathcal{L}_{1}$ introduced in $\S 2$ of that paper will still denote the set of block maps $f$ for which $Q f=1$, i.e., block maps of the form $f=I+T R f$. It follows from [1] (2.3) that if $\phi$ is linear in the first $s-1$ variables then

$$
\begin{aligned}
\phi & =a_{1} I+\cdots+a_{s-1} T^{s-2} I+T^{s-1} R_{s-1} \phi \\
& =a_{1} I+\cdots+a_{s-1} T^{s-2} I+T^{s-1} I \cdot T^{s} Q_{s} \phi+T^{s} R_{s} \phi,
\end{aligned}
$$

and given $s$ these expressions are unique.

A block map is said to be linear if it is linear in all its variables. With the operations of addition and composition, the set of all block maps $\mathscr{F}$ satisfies all the axioms for a ring except the right distributive law. An example is given in [1] for which $h \circ(f+g) \neq h \circ f+h \circ g$. However, the following is true.

(1.2) Lemma. If $h$ is linear, i.e., $h=a_{0}+\sum_{i=1}^{n} a_{i} T^{i-1} I$, then $h \circ(f+g)$ $=h \circ f+h \circ g+a_{0}$.

(1.3) LemMa. If $h$ is linear and not constant then $h \circ f \simeq h \circ g$ implies $f \simeq g$.

Proof. Let $h \circ f+h \circ g \in Z_{2}$. Then by (1.2), $h \circ(f+g) \in Z_{2}$ so that $\rho[h \circ(f+g)]=0$. If $\rho(f+g)>0$, the conditions on $h$ ensure that $\rho[h \circ(f+g)]=\rho(h)+\rho(f+g)-1>0$. The contradiction proves that $f+g \in Z_{2}$.

(1.4) Lemma. Let $\phi$ be linear in the first $r-1$ variables and $\psi$ be linear in the first $s-1$ variables with $r \geqslant 2$ and $s \geqslant 1$. If $r \geqslant s$ then $\phi \circ \psi$ and $\psi \circ \phi$ are linear in the first $s-1$ variables. If $r>s$ then

$$
Q_{s}(\phi \circ \psi) \simeq Q_{1} \phi \cdot Q_{s} \psi, \quad Q_{s}(\psi \circ \phi) \simeq Q_{1} \phi\left(Q_{s} \psi \circ \phi\right) .
$$

If $r=s$ then

$$
Q_{s}(\phi \circ \psi) \simeq Q_{1} \phi \cdot Q_{s} \psi+Q_{1} \psi\left(Q_{s} \phi \circ \psi\right)
$$

Proof. Let

$$
\phi=a_{1} I+\cdots+a_{r-1} T^{r-2} I+T^{r-1} R_{r-1} \phi
$$

and

$$
\psi=b_{1} I+\cdots+b_{s-1} T^{s-2} I+T^{s-1} R_{s-1} \psi
$$

Then

$$
\phi \circ \psi=a_{1} \psi+\cdots+a_{r-1} T^{r-2} \psi+T^{r-1} R_{r-1} \phi \circ \psi
$$

Thus

$$
Q_{i}(\phi \circ \psi)=a_{1} Q_{i} \psi+\cdots+a_{r-1} Q_{i} T^{r-2} \psi+Q_{i}\left(T^{r-1} R_{r-1} \phi \circ \psi\right) \in Z_{2}
$$

if $i \leqslant s-1 \leqslant r-1$. If $s=r$

$$
Q_{s}(\phi \circ \psi)=a_{1} Q_{s} \psi+a_{2} b_{s-1}+\cdots+a_{s-1} b_{2}+Q_{1}\left(R_{s-1} \phi \circ \psi\right) .
$$

By [1] (2.9)* we have

$$
Q_{1}\left(R_{s-1} \phi \circ \psi\right)=Q_{1} \psi\left(Q_{1} R_{s-1} \phi \circ \psi\right) .
$$


Thus if $s=r, Q_{s}(\phi \circ \psi) \simeq Q_{1} \phi \cdot Q_{s} \psi+Q_{1} \psi\left(Q_{s} \phi \circ \psi\right)$. If $s<r$ then $Q_{s}\left(T^{r-1} R_{r-1} \phi \circ \psi\right)=0$ and $Q_{s}(\phi \circ \psi) \simeq Q_{1} \phi \cdot Q_{s} \psi$. Also

$$
\psi \circ \phi=b_{1} \phi+\cdots+b_{s-1} T^{s-2} \phi+T^{s-1} R_{s-1} \psi \circ \phi
$$

and

$$
Q_{i}(\psi \circ \phi)=b_{1} Q_{i} \phi+\cdots+b_{s-1} Q_{i} T^{s-2} \phi+Q_{i}\left(T^{s-1} R_{s-1} \psi \circ \phi\right) \in Z_{2}
$$

if $i \leqslant s-1 \leqslant r-1$. If $s<r$ then

$$
Q_{s}(\psi \circ \phi) \simeq Q_{s}\left(T^{s-1} R_{s-1} \psi \circ \phi\right) \simeq Q_{1} \phi\left(Q_{s} \psi \circ \phi\right) .
$$

(1.5) Lemma. Suppose $f \in \mathcal{L}_{1}$ and $R f$ is nonconstant and is linear in the first $s-1$ variables. If $\phi+\phi \circ f$ is linear in the first $t-1$ variables, and $t \leqslant s$, then $\phi$ is linear in the first $t-1$ variables.

Proof. If $\phi+\phi \circ f$ is linear in one variable then $Q_{1} \phi+Q_{1}(\phi \circ f)=Q_{1} \phi+$ $Q_{1} \phi \circ f \in Z_{2}$. Thus by [1] $(2.11)^{*} Q_{1} \phi \in Z_{2}$. Suppose $\phi$ is linear in the first $r-1$ variables with $r<t$, i.e., $\phi=b_{1} I+\cdots+b_{r-1} T^{r-2} I+T^{r-1} R_{r-1} \phi$. Then

$$
Q_{r} \phi+Q_{r}(\phi \circ f) \simeq Q_{r} \phi+Q_{r} \phi \circ f \in Z_{2} \text {. }
$$

Hence $Q_{r} \phi \in Z_{2}$. It follows that $\phi$ is linear in the first $t-1$ variables.

(1.6) LEMMA. Suppose $f, g \in \mathcal{L}_{1}$, and $R f$ and $R g$ are nonconstant and linear in the first $s-1$ variables. If $\phi$ is linear in the first $t-1$ variables, and $t \leqslant s$, and

$$
\phi+\phi \circ g+\psi+\psi \circ f \in Z_{2}
$$

then $\psi$ is linear in the first $t-1$ variables and

$$
Q_{t} \phi+Q_{t} \phi \circ g+Q_{t} \psi+Q_{t} \psi \circ f \in Z_{2} .
$$

If also $\phi$ is not linear in the first $t$ variables then $\psi$ is not linear in the first $t$ variables.

Proof. By [1] (2.9)* we have

$$
Q_{1} \phi+Q_{1} \phi \circ g+Q_{1} \psi+Q_{1} \psi \circ f \in Z_{2} \text {. }
$$

Then $Q_{1} \phi \in Z_{2}$ implies $Q_{1} \phi+Q_{1} \phi \circ g=0$ so that $Q_{1} \psi+Q_{1} \psi \circ f \in Z_{2}$ and $Q_{1} \psi \in Z_{2}$. Suppose $t-1 \geqslant 1$ and $\psi$ is linear in the first $r-1$ variables where $r<t$. Then

$$
Q_{r} \phi+Q_{r} \phi \circ g+Q_{r} \psi+Q_{r} \psi \circ f \in Z_{2} \text {. }
$$

Since $Q_{r} \phi+Q_{r} \phi \circ g \in Z_{2}$, it follows again that $Q_{r} \psi \in Z_{2}$. Thus $\psi$ is linear in the first $t-1$ variables and

$$
Q_{t} \phi+Q_{t} \phi \circ g+Q_{t} \psi+Q_{t} \psi \circ f \in Z_{2} \text {. }
$$

Again $Q_{t} \phi \in Z_{2}$ if and only if $Q_{t} \psi \in Z_{2}$.

(1.7) Lemma. Suppose $f, g \in \mathcal{L}_{1}$, and $R f$ and $R g$ are linear in the first $s-1$ variables, and that $\rho\left(Q_{s} R g\right)>\rho\left(Q_{s} R f\right)>0$. If $\phi$ is linear in the first $r-1$ variables but not the first $r$ variables for some $r>s$ and

$$
\phi+\phi \circ g+\psi+\psi \circ f \in Z_{2}
$$

then $\psi$ is linear in the first $t-1$ but not the first $t$ variables for some $t$ such that $s<t \leqslant r$. 
Proof. Since $\phi$ is certainly linear in the first $s-1$ variables, it follows from (1.6) that $\psi$ is also linear in the first $s-1$ variables and

$$
Q_{s} \phi+Q_{s} \phi \circ g+Q_{s} \psi+Q_{s} \psi \circ f \in Z_{2} \text {. }
$$

Now $Q_{s} \phi$ is also constant, so $Q_{s} \psi+Q_{s} \psi \circ f \in Z_{2}$ and $Q_{s} \psi \in Z_{2}$. Thus, by Lemma (1.4)

$$
\begin{aligned}
Q_{s+1} \phi+Q_{s+1}(\phi \circ g) & +Q_{s+1} \psi+Q_{s+1}(\psi \circ f) \\
= & Q_{s+1} \phi+Q_{s+1} \phi \circ g+Q_{1} \phi \cdot Q_{s+1} g \\
& +Q_{s+1} \psi+Q_{s+1} \psi \circ f+Q_{1} \psi \cdot Q_{s+1} f \in Z_{2} .
\end{aligned}
$$

Two cases are easily dealt with. If $Q_{1} \phi=1$ and $\rho\left(Q_{s+1} \phi\right)=0$, then

$$
\rho\left(Q_{s+1} \psi+Q_{s+1} \psi \circ f+Q_{1} \psi \cdot Q_{s+1} f\right)=\rho\left(Q_{s+1} g\right)>0 \text {. }
$$

The assumption that $\rho\left(Q_{s+1} \psi\right)=0$ leads to the contradiction $\rho\left(Q_{1} \psi \cdot Q_{s+1} f\right)=$ $\rho\left(Q_{s+1} g\right)>\rho\left(Q_{s+1} f\right)$. If $\rho\left(Q_{s+1} \phi\right)>0$, then

$$
\rho\left(Q_{s+1} \psi+Q_{s+1} \psi \circ f+Q_{1} \psi \cdot Q_{s+1} f\right)=\rho\left(Q_{s+1} \phi\right)+\rho(g)-1 .
$$

The assumption that $\rho\left(Q_{s+1} \psi\right)=0$ leads to the contradiction

$$
\rho\left(Q_{1} \psi \cdot Q_{s+1} f\right)=\rho\left(Q_{s+1} \phi\right)+\rho(g)-1>\rho\left(Q_{s+1} g\right)>\rho\left(Q_{s+1} f\right) .
$$

Thus in both these cases $\psi$ is linear in the first $s$ variables but not the first $s+1$ variables.

In the remaining case $Q_{1} \phi=0$ and $\rho\left(Q_{s+1} \phi\right)=0$, so that

If $\rho\left(Q_{s+1} \psi\right)>0$ then

$$
Q_{s+1} \psi+Q_{s+1} \psi \circ f+Q_{1} \psi \cdot Q_{s+1} f \in Z_{2} \text {. }
$$

$$
\begin{aligned}
\rho\left(Q_{s+1} \psi \circ f\right) & =\rho\left(Q_{s+1} \psi\right)+\rho(f)-1=\rho\left(Q_{s+1} \psi+Q_{1} \psi \cdot Q_{s+1} f\right) \\
& \leqslant \max \left\{\rho\left(Q_{s+1} \psi\right), \rho\left(Q_{s+1} f\right)\right\} .
\end{aligned}
$$

The contradiction shows that $\rho\left(Q_{s+1} \psi\right)=0$ and consequently $Q_{1} \psi=0$. It follows that $\phi=T R \phi$ and $\psi=T R \psi$, and that

$$
R \phi+R \phi \circ g+R \psi+R \psi \circ f \in Z_{2} \text {. }
$$

Since $R \phi$ and $R \psi$ are linear in the first $s$ variables,

$$
Q_{s+1} R \phi+Q_{s+1}(R \phi \circ g)+Q_{s+1} R \psi+Q_{s+1}(R \psi \circ f) \in Z_{2} .
$$

We may now apply the three cases to $R \phi$ and $R \psi$. If $Q_{1} R \phi=1$ and $\rho\left(Q_{s+1} R \phi\right)=0$, or if $\rho\left(Q_{s+1} R \phi\right)>0$, then $\rho\left(Q_{s+1} R \psi\right)=\rho\left(Q_{s+2} \psi\right)>0$, so that $\psi$ is linear in the first $s+1$ variables but not the first $s+2$ variables. If $Q_{1} R \phi=0$ and $\rho\left(Q_{s+1} R \phi\right)=0$, then $\rho\left(Q_{s+1} R \psi\right)=0$ so that $\phi$ and $\psi$ are linear in the first $s+2$ variables, and the argument can be repeated. The assumption that $\rho\left(Q_{r} \phi\right)>0$ for some $r>s$ guarantees that for some $t$ for which $s<t \leqslant r$ one of the first two cases applies to $Q_{s+1} R_{t-s-1} \phi=Q_{t} \phi$ and that $\psi$ is linear in the first $t-1$ but not the first $t$ variables.

2. A property of powers of block maps. A block map $\phi$ will be said to be linear relative to $r_{1}, r_{2}, \ldots, r_{n}$ if $\phi$ is linear in the first $r_{1}-1$ but not in the first $r_{1}$ variables, and for each $i$ for which $1 \leqslant i<n$ the quotient $Q_{r_{i}} \ldots Q_{r_{2}} Q_{r_{1}} \phi$ is linear in the first $r_{i+1}-1$ but not the first $r_{i+1}$ variables. Note that $r_{i} \geqslant 1$ for $1 \leqslant i \leqslant n$. 
For each nonlinear block map $\phi$ there is a unique sequence $r_{1}, r_{2}, \ldots, r_{n}$ such that $\phi$ is linear relative to that sequence and $Q_{r_{n}} \ldots Q_{r_{2}} Q_{r_{1}} \phi$ is a nonconstant linear block map. In this paper I study the property that $r_{i} \leqslant r_{1}$ for all $i$ such that $2 \leqslant i \leqslant n$ (or $n=1)$. If $f \in \mathcal{L}_{1}$, let $C^{*}(f)=\left\{g \in \mathfrak{L}_{1} \mid g \circ f=f \circ g\right\}$.

(2.1) Proposition. Suppose $f \in \mathfrak{L}_{1}$, and $R f$ is linear relative to $r_{1}, r_{2}, \ldots, r_{n}$ where $r_{i} \leqslant r_{1}$ for $2 \leqslant i \leqslant n($ or $n=1)$. If $g \in C^{*} f$ and $\mathrm{Rg}$ is nonconstant, then $\mathrm{Rg}$ is linear relative to $r_{1}, r_{2}, \ldots, r_{n}$. Moreover, for $1 \leqslant \mu \leqslant n$,

$$
\begin{aligned}
Q_{r_{\mu}} \ldots Q_{r_{2}} Q_{r_{1}} R g+ & Q_{r_{\mu}} \ldots Q_{r_{2}} Q_{r_{1}} R g \circ f \\
& +Q_{r_{\mu}} \ldots Q_{r_{2}} Q_{r_{1}} R f+Q_{r_{\mu}} \ldots Q_{r_{2}} Q_{r_{1}} R f \circ g \in Z_{2} .
\end{aligned}
$$

Proof. Certainly $g \in \mathfrak{L}_{1}$, and

$$
R g+R g \circ f+R f+R f \circ g=0 .
$$

Now $R f$ is linear in the first variable if and only if $Q_{1} R f \in Z_{2}$ which occurs if and only if $Q_{1} R g \in Z_{2}$. Suppose $R g$ is linear in the first $i-1$ variables with $i<r_{1}$. Then $R f \circ g$ is linear in the first $i$ variables. So by (1.5) $R g$ is linear in the first $i$ variables. Hence $R g$ is linear in the first $r_{1}-1$ variables and

$$
Q_{r_{1}} R g+Q_{r_{1}} R g \circ f+Q_{r_{1}} R f+Q_{r_{1}} R f \circ g \in Z_{2} .
$$

Since $\rho\left(Q_{r_{1}} R f\right)>0$ and $\rho(g) \geqslant 2$ we have $\rho\left(Q_{r_{1}} R g\right)>0$. Thus $R g$ is linear relative to $r_{1}$. Suppose that $R g$ is linear relative to $r_{1}, r_{2}, \ldots, r_{\mu}$, where $\mu<n$, and suppose that

$$
\begin{aligned}
Q_{r_{\mu}} \ldots Q_{r_{2}} Q_{r_{1}} R g+ & Q_{r_{\mu}} \ldots Q_{r_{2}} Q_{r_{1}} R g \circ f \\
& +Q_{r_{\mu}} \ldots Q_{r_{2}} Q_{r_{1}} R f+Q_{r_{\mu}} \ldots Q_{r_{2}} Q_{r_{1}} R f \circ g \in Z_{2} .
\end{aligned}
$$

By (1.6), $Q_{r_{\mu}} \ldots Q_{r_{2}} Q_{r_{1}} R g$ is linear in the first $r_{\mu+1}-1$ but not the first $r_{\mu+1}$ variables and $\mu$ can be replaced by $\mu+1$ in the inductive assumptions. Thus $R g$ is linear relative to $r_{1}, r_{2}, \ldots, r_{n}$.

(2.2) Proposition. Suppose $f \in \mathcal{L}_{1}$, and $R f$ is linear relative to $r_{1}, r_{2}, \ldots, r_{n}$ where $r_{i} \leqslant r_{1}$ for $2 \leqslant i \leqslant n-1$ and $r_{n}>r_{1}\left(\right.$ or $n=2$ and $\left.r_{2}>r_{1}\right)$. Then for each $\nu \geqslant 2, R f^{\nu}$ is linear relative to $r_{1}, r_{2}, \ldots, r_{n-1}, s_{n}$ with $r_{1}<s_{n} \leqslant r_{n}$.

Proof. Since $f^{\nu} \in C^{*}(f)$, it follows from (2.1) that $R f^{\nu}$ is linear relative to $r_{1}, r_{2}, \ldots, r_{n-1}$. Putting $\mu=1$ and $\mu=n-1$ in the final conclusion of (2.1) we obtain

$$
Q_{r_{1}} R f^{\nu}+Q_{r_{1}} R f^{\nu} \circ f+Q_{r_{1}} R f+Q_{r_{1}} R f \circ f^{\nu} \in Z_{2}
$$

and

$$
\begin{aligned}
Q_{r_{n-1}} \ldots Q_{r_{2}} Q_{r_{1}} R f^{\nu} & +Q_{r_{n-1}} \ldots Q_{r_{2}} Q_{r_{1}} R f^{\nu \circ f} \\
& +Q_{r_{n-1}} \ldots Q_{r_{2}} Q_{r_{1}} R f+Q_{r_{n-1}} \ldots Q_{r_{2}} Q_{r_{1}} R f \circ f^{\nu} \in Z_{2} .
\end{aligned}
$$

The first of these gives

$$
\rho\left(Q_{r_{1}} R f^{\nu}+Q_{r_{1}} R f^{\nu} \circ f\right)=\rho\left(Q_{r_{1}} R f+Q_{r_{1}} R f \circ f^{\nu}\right)
$$


and so

$$
\rho\left(Q_{r_{1}} R f^{\nu}\right)+\rho(f)-1=\rho\left(Q_{r_{1}} R f\right)+\rho\left(f^{\nu}\right)-1 \text {. }
$$

Thus

$$
\rho\left(Q_{r_{1}} R f^{\nu}\right)+\rho(R f)=\rho\left(Q_{r_{1}} R f\right)+\nu \rho(R f)
$$

and

$$
\rho\left(Q_{r_{1}} R f^{\nu}\right)=\rho\left(Q_{r_{1}} R f\right)+(\nu-1) \rho(R f)>\rho\left(Q_{r_{1}} R f\right)>0 .
$$

The result then follows from (1.7) with

$$
Q_{r_{n-1}} \ldots Q_{r_{2}} Q_{r_{1}} R f=\phi \text { and } Q_{r_{n-1}} \ldots Q_{r_{2}} Q_{r_{1}} R f^{\nu}=\psi \text {. }
$$

(2.3) Notation. For the rest of this section and the next $f \in \mathcal{L}_{1}$ is a function with $\rho(R f)=p$ and $R f$ is linear relative to $r_{1}, r_{2}, \ldots, r_{n}$ with $r_{1} \geqslant 1$ and $r_{i} \leqslant r_{1}$ for $1 \leqslant i \leqslant n$ (or $n=1$ ). The operator $Q_{r_{n}} \ldots Q_{r_{2}} Q_{r_{1}}$ will be abbreviated to 2 .

It is assumed also that $2 R f=T^{l-1} I+T^{l} \theta, l \geqslant 1$, where $\theta$ is linear and $\rho(\mathcal{Q} R f)=m \geqslant l$.

Given such a block map $f$, we define an operator $L$ on the set of block maps as follows:

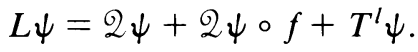

The operator $L$ is additive.

(2.4) Proposition. If $g \in C^{*}(f)$ and $R g$ is nonconstant then $R g$ is linear relative to $r_{1}, r_{2}, \ldots, r_{n}$ and $L R g+T^{l+1} \theta \circ R g \in Z_{2}$.

Proof. By (2.1) we have

$$
2 R g+2 R g \circ f+2 R f+2 R f \circ g \in Z_{2} .
$$

Also by (1.2)

$$
2 R f+2 R f \circ g \simeq 2 R f \circ T R g=T^{l} R g+T^{l+1} \theta \circ R g
$$

(2.5) Proposition. Suppose $L \psi+T^{l+1} \theta \circ \psi \in Z_{2}$ and $\psi$ is linear relative to $r_{1}, r_{2}, \ldots, r_{n}$. Then $2 \psi=a T^{l-1} I+T^{l} \psi^{\prime}\left(\right.$ with $a \in Z_{2}$ and $\left.\psi^{\prime}=R^{\prime} \mathcal{2} \psi\right)$ and $\psi^{\prime}$ is linear in the first $r_{1}-1$ variables. Also

$$
Q_{r_{1}} \psi^{\prime}+Q_{r_{1}} \psi^{\prime} \circ f+a Q_{r_{1}} R f+Q_{r_{1}} \psi \in Z_{2} \text {. }
$$

Moreover, $\psi^{\prime}$ is linear in the first $r_{1}$ variables if and only if $a=1$ and $Q_{r_{1}} R f \simeq Q_{r_{1}} \psi$.

Proof. Since

$$
Q_{1} \mathcal{2} \psi+Q_{1} \mathcal{Q} \psi \circ f+Q_{1} T^{l} \psi+Q_{1} T^{l+1} \theta \circ \psi=Q_{1} \mathcal{L} \psi+Q_{1} \mathcal{2} \psi \circ f \in Z_{2},
$$

we have $Q_{1} \mathscr{Q} \psi \in Z_{2}$. Thus $2 \psi=c_{0} I+T R \mathscr{L} \psi$ for some $c_{0} \in Z_{2}$, and

$$
c_{0} I+T R \mathscr{Q} \psi+c_{0}(I+T R f)+T R \mathscr{2} \psi \circ f+T^{l} \psi+T^{l+1} \theta \circ \psi \in Z_{2} .
$$

If $l=1$, then with $c_{0}=a$ and $\psi^{\prime}=R \mathscr{2} \psi$ we have $\psi^{\prime}+\psi^{\prime} \circ f+\psi+a R f+$ $T \theta \circ \psi \in Z_{2}$. Since $\psi+a R f+T \theta \circ \psi$ is linear in the first $r_{1}-1$ variables, so also is 
$\psi^{\prime}+\psi^{\prime} \circ f$. Thus by (1.5), $\psi^{\prime}$ is linear in the first $r_{1}-1$ variables. Hence $Q_{r_{1}}\left(\psi^{\prime} \circ f\right)$ $=Q_{r_{1}} \psi^{\prime} \circ f$. Since also $Q_{r_{1}}(T \theta \circ \psi) \in Z_{2}$ we have

$$
Q_{r_{1}} \psi^{\prime}+Q_{r_{1}} \psi^{\prime} \circ f+Q_{r_{1}} \psi+a Q_{r_{1}} R f \in Z_{2} \text {. }
$$

If $l>1$, then

$$
R \mathscr{2} \psi+R \mathscr{2} \psi \circ f+c_{0} R f+T^{l-1} \psi+T^{\prime} \theta \circ \psi \in Z_{2} .
$$

Since $c_{0} R f+T^{l-1} \psi+T^{l} \theta \circ \psi$ is linear in the first $r_{1}-1$ variables, so also is $R \mathscr{2} \psi$ and

$$
Q_{r_{1}} R \mathcal{2} \psi+Q_{r_{1}} R \mathcal{2} \psi \circ f+c_{0} Q_{r_{1}} R f \in Z_{2} .
$$

Now the assumption $\rho\left(Q_{r_{1}} R \mathcal{2} \psi\right)>0$ leads to the contradiction

$$
\rho\left(Q_{r_{1}} R \mathcal{2} \psi\right)+\rho(R f)=\rho\left(c_{0} Q_{r_{1}} R f\right)<\rho(R f) .
$$

Thus $\rho\left(Q_{r_{1}} R \mathscr{2} \psi\right)=0$, and so $c_{0}=0$. Hence $2 \psi=T R \mathscr{2} \psi$ and

$$
R \mathscr{2} \psi+R 2 \psi \circ f+T^{l-1} \psi+T^{l} \theta \circ \psi \in Z_{2} .
$$

We prove inductively that if $s<l$ then $2 \psi=T^{s} R^{s} 2 \psi$. Suppose $2 \psi=T^{s-1} R^{s-1} 2 \psi$. Then $R^{s-1} 2 \psi+R^{s-1} 2 \psi \circ f+T^{l-s+1} \psi+T^{l-s+2} \theta \circ \psi \in Z_{2}$. Hence $Q_{1} R^{s-1} 2 \psi+Q_{1} R^{s-1} 2 \psi \circ f \in Z_{2}$ and so $Q_{1} R^{s-1} \mathcal{L} \psi \in Z_{2}$. Thus $R^{s-1} \mathcal{Q} \psi=c_{s-1} I+T R^{s} \mathcal{Q} \psi$ for some $c_{s-1} \in Z_{2}$ and

$$
\begin{aligned}
& c_{s-1} I+T R^{s} \mathcal{Q} \psi+c_{s-1}(I+T R f)+T R^{s} \mathcal{Q} \psi \circ f \\
&+T^{l-s+1} \psi+T^{l-s+2} \theta \circ \psi \in Z_{2} .
\end{aligned}
$$

Since $c_{s-1} R f+T^{l-s} \psi+T^{l-s+1} \theta \circ \psi$ is linear in the first $r_{1}-1$ variables, so also is $R^{s} \mathcal{Q} \psi$ and

$$
Q_{r_{1}} R^{s} \mathcal{Q} \psi+Q_{r_{1}} R^{s} \mathcal{Q} \psi \circ f+c_{s-1} Q_{r_{1}} R f \in Z_{2} .
$$

Now $\rho\left(Q_{r_{1}} R^{s} \mathcal{Q} \psi\right)>0$ leads to a contradiction on spans, while $\rho\left(Q_{r_{1}} R^{s} \mathcal{Q} \psi\right)=0$ implies $c_{s-1}=0$ and $R^{s-1} \mathcal{Q} \psi=T R^{s} \mathcal{Q} \psi$, so that $2 \psi=T^{s} R^{s} \mathcal{Q} \psi$. It follows that $2 \psi=T^{l-1} R^{l-1} 2 \psi$. As before we have $R^{l-1} \mathcal{2} \psi=c_{l-1} I+T R^{l} \mathcal{L} \psi$ for some $c_{l-1} \in Z_{2}$, and

$$
R^{\prime} \mathscr{Q} \psi+R^{\prime} \mathscr{Q} \psi \circ f+c_{l-1} R f+\psi+T \theta \circ \psi \in Z_{2} .
$$

Thus with $c_{l-1}=a$ and $R^{\prime} \mathscr{Q} \psi=\psi^{\prime}$,

$$
Q_{r_{1}} \psi^{\prime}+Q_{r_{1}} \psi^{\prime} \circ f+a Q_{r_{1}} R f+Q_{r_{1}} \psi \in Z_{2} \text {. }
$$

Finally, $Q_{r_{1}} \psi^{\prime} \in Z_{2}$ implies $\rho\left(a Q_{r_{1}} R f+Q_{r_{1}} \psi\right)=0$. Since $\rho\left(Q_{r_{1}} \psi\right)>0$, it then follows that $a=1$ and $Q_{r_{1}} R f \simeq Q_{r_{1}} \psi$.

(2.6) Theorem. Suppose $g \in \mathfrak{L}_{1}$, and $R g$ is nonlinear. Then for each $\nu \geqslant 2, R g^{\nu}$ is linear relative to $s_{1}, s_{2}, \ldots, s_{n+1}$ with $s_{n+1}>s_{1}$ for some $n$.

Proof. Proposition (2.2) deals with the case when $R g$ is linear relative to $s_{1}, s_{2}, \ldots, s_{m}$ with $s_{m}>s_{1}$ for some $m$. All other block maps $f \in \mathcal{L}_{1}$, with $R f$ nonconstant, are of the type studied in (2.4) and (2.5). For such maps

$$
2 R f^{\nu}=a T^{l-1} I+T^{l} \psi^{\prime}
$$


with $\psi^{\prime}$ linear in the first $r_{1}-1$ variables, and

$$
Q_{r_{1}} \psi^{\prime}+Q_{r_{1}} \psi^{\prime} \circ f+a Q_{r_{1}} R f+Q_{r_{1}} R f^{\nu} \in Z_{2} \text {. }
$$

By the same arguments as those used in the proof of (2.1)

$$
\rho\left(Q_{r_{1}} R f^{\nu}\right)+\rho(R f)=\rho\left(Q_{r_{1}} R f\right)+\rho\left(R f^{\nu}\right)=\rho\left(Q_{r_{1}} R f\right)+\nu \rho(R f) .
$$

Thus $\rho\left(a Q_{r_{1}} R f+Q_{r_{1}} R f^{\nu}\right)>0$ and so $\rho\left(Q_{r_{1}} \psi^{\prime}\right)>0$. Since $\psi^{\prime}$ is linear in the first $r_{1}-1$ but not the first $r_{1}$ variables, $2 R f^{v}$ is linear in the first $r_{1}+l-1$ but not the first $r_{1}+l$ variables. Thus $R f^{v}$ is linear relative to $r_{1}, r_{2}, \ldots, r_{n}, r_{1}+l$ with $r_{1}+l>r_{1}$.

3. The sums of powers theorem. In view of Theorem (2.6), a block map $f$ for which $R f$ is linear relative to $r_{1}, r_{2}, \ldots, r_{n}$ with $r_{i} \leqslant r_{1}$ for $2 \leqslant i \leqslant n$ (or $n=1$ ) is not a power of any other block map. We now show that such a block map commutes at most with sums of odd numbers of its powers.

(3.1) Proposition. Suppose $L \psi+b T^{l} \theta+c T^{l+1} \theta \circ \psi \in Z_{2}, b, c \in Z_{2}$, and $\psi$ is linear relative to $r_{1}, r_{2}, \ldots, r_{n}$. Then $2 \psi=a T^{l-1} I+T^{l} \psi^{\prime}$ (with $a \in Z_{2}$ and $\left.\psi^{\prime}=R^{\prime} \mathscr{Q} \psi\right)$. Moreover, $\psi^{\prime}$ is linear relative to $r_{1}, r_{2}, \ldots, r_{n}$ and $L \psi^{\prime}+a T^{\prime} \theta \in Z_{2}$. If $\rho\left(\mathcal{2} \psi^{\prime}\right)=0$ and $\rho(\mathcal{2} \psi)>0$ then $a=1$ and $\mathcal{2} \psi \simeq \mathscr{2} R$. If $\rho\left(\mathcal{2} \psi^{\prime}\right)>0$ then $\rho\left(\mathcal{2} \psi^{\prime}\right)=\rho(\mathcal{L} \psi)-p$.

Proof. The proof of Proposition (2.5) needs little modification to show that in this case also

$$
Q_{r_{1}} \psi^{\prime}+Q_{r_{1}} \psi^{\prime} \circ f+a Q_{r_{1}} R f+Q_{r_{1}} \psi \in Z_{2} .
$$

Now $a Q_{r_{1}} R f+Q_{r_{1}} \psi$ is linear relative to $r_{2}, \ldots, r_{n}$ (if $n>1$ ). Thus so also is $Q_{r_{1}} \psi^{\prime}+Q_{r_{1}} \psi^{\prime} \circ f$, and by repeated application of Lemma (1.5) $Q_{r_{1}} \psi^{\prime}$ is linear relative to $r_{2}, r_{3}, \ldots, r_{n}$. Thus

$$
\begin{aligned}
2 \psi^{\prime}+2 \psi^{\prime} & \circ f+a 2 R f+2 \psi \\
& =2 \psi^{\prime}+2 \psi^{\prime} \circ f+a\left(T^{l-1} I+T^{\prime} \theta\right)+a T^{l-1} I+T^{\prime} \psi^{\prime} \\
& =L \psi^{\prime}+a T^{\prime} \theta \in Z_{2} .
\end{aligned}
$$

If $\rho\left(2 \psi^{\prime}\right)=0$ then $a \mathscr{2} R f+\mathscr{2} \psi \in Z_{2}$. Thus if also $\rho(\mathcal{2} \psi)>0$ then $a=1$ and $2 \psi \simeq 2 R f$. On the other hand, if $\rho\left(2 \psi^{\prime}\right)>0$ then

$$
\rho\left(2 \psi^{\prime}+2 \psi^{\prime} \circ f+a 2 R f\right)=\rho\left(2 \psi^{\prime}\right)+p=\rho(2 \psi) .
$$

(3.2) Notation. Let $\psi$ be a block map which is linear relative to $r_{1}, r_{2}, \ldots, r_{n}$ and for which $L \psi+T^{l+1} \theta \circ \psi \in Z_{2}$. Associate with $\psi$ a sequence of constants $a_{0}, a_{1}, a_{2}, \ldots$ by repeated application of Proposition (3.1). Let $\psi=\psi_{0}$ and for $i>0$ let $2 \psi_{i}=a_{i} T^{l-1} I+T^{l} \psi_{i+1}$.

Note that for large enough $i$ both $a_{i}=0$ and $\psi_{i}=0$. Note also that if $L \psi+$ $T^{l+1} \theta \circ \psi \in Z_{2}$ and $L \phi+T^{l+1} \theta \circ \phi \in Z_{2}$ then $L(\psi+\phi)+T^{l+1} \theta \circ(\psi+\phi) \in Z_{2}$ and $a_{i}(\psi+\phi)=a_{i}(\psi)+a_{i}(\phi)$ for all $i \geqslant 0$.

(3.3) Proposition. Suppose $L \psi+T^{l+1} \theta \circ \psi \in Z_{2}$ and $\psi$ is linear relative to $r_{1}$, $r_{2}, \ldots, r_{n}$. If $\rho(\psi)>0$ then $\rho(\psi)=$ tp for some $t \geqslant 1$. If $t=1$ then $\psi \simeq R$. If $t>1$ then $a_{t-1}(\psi)=1$. 
Proof. If $\rho(\mathcal{2} \psi)=0$ then $T^{l} \psi+T^{l+1} \theta \circ \psi \in Z_{2}$ so that $\rho(\psi)=0$. Hence $\rho(\mathcal{Q} \psi)>0$ and

$$
\rho\left(T^{l} \psi+T^{l+1} \theta \circ \psi\right)=\rho(\mathscr{2} \psi+2 \psi \circ f)
$$

so that $m+\rho(\psi)=\rho(\mathscr{2} \psi)+p$. Moreover, if $\rho\left(\mathcal{2} \psi_{1}\right)=0$ then Proposition (3.1) ensures that $a_{0}=1$ and $\mathcal{L} \psi \simeq \mathscr{2} R f$. Then

$$
L \psi+T^{l+1} \theta \circ \psi=2 R f+2 R f \circ f+T^{l} \psi+T^{l+1} \theta \circ \psi \in Z_{2},
$$

i.e.,

$$
\left(T^{l-1} I+T^{l} \theta\right) \circ(I+f)+\left(T^{l-1} I+T^{l} \theta\right) \circ T \psi \in Z_{2} .
$$

Thus from Lemma (1.3) we have $R f \simeq \psi$ and $\rho(\psi)=p$.

However, if $\rho\left(\mathcal{2} \psi_{1}\right)>0$ then there exists $t \geqslant 2$ such that $\rho\left(\mathcal{2} \psi_{t-1}\right)>0$ and $\rho\left(2 \psi_{t}\right)=0$. Then $a_{t-1}=1$ and $\rho\left(2 \psi_{t-1}\right)=\rho(2 R f)=m$. But Proposition (3.1) also ensures that $\rho\left(2 \psi_{t-1}\right)=\rho\left(2 \psi_{t-2}\right)-p=\rho\left(2 \psi_{0}\right)-(t-1) p$. Thus $\rho\left(2 \psi_{t-1}\right)=\rho(\psi)+m-p-(t-1) p$ and $\rho(\psi)=t p$.

(3.4) Proposition. If $L \psi+T^{l+1} \theta \circ \psi \in Z_{2}$, and $\psi$ is linear relative to $r_{1}, r_{2}, \ldots, r_{n}$, then $\psi$ differs from a sum of remainders of powers of $f$ by at most a constant.

Proof. Proposition (3.3) ensures that $\rho(\psi)=t p$ and if $t=1$ then $\psi \simeq R f$. Now let $t>1$. Then by Proposition (2.4) $L R f^{t}+T^{l+1} \theta \circ R f^{t} \in Z_{2}$ and $L\left(\psi+R f^{t}\right)+$ $T^{l+1} \theta \circ\left(\psi+R f^{t}\right) \in Z_{2}$. Since $\rho\left(\psi+R f^{t}\right) \leqslant \max \left\{\rho(\psi), \rho\left(R f^{t}\right)\right\}=t p$ we have $\rho\left(\psi+R f^{t}\right)=s p$ with $s \leqslant t$. But $a_{t-1}\left(\psi+R f^{t}\right)=a_{t-1} \psi+a_{t-1} R f^{t}=1+1=0$, so that $\rho\left(\psi+R f^{t}\right) \neq t p$. If we assume inductively that the result holds for $\psi$ with $\rho(\psi)=s p, s<t$, then we obtain the result for $\psi$ with $\rho(\psi)=t p$.

(3.5) THEOREM. Suppose $f \in \mathcal{L}_{1}$ and $R f$ is linear relative to $r_{1}, r_{2}, \ldots, r_{n}$ with $r_{1} \geqslant 1$ and $r_{i} \leqslant r_{1}$ for $1 \leqslant i \leqslant n($ or $n=1)$, and $Q_{r_{n}} \ldots Q_{r_{2}} Q_{r_{1}} R f$ is a nonconstant linear map. Then every member of $C^{*}(f)$ differs from the sum of an odd number of powers of $f$ by at most a constant.

Proof. Suppose $g \in C^{*}(f)$. Then $g=f^{0}+T R g$, and Propositions (2.4) and (3.4) ensure that $R g$ differs from a sum of remainders of powers of $f$ by at most a constant. Since $T R f^{n}=f^{0}+f^{n}$, if $R g$ is the sum of $m$ remainders of powers of $f$ then $g$ is the sum of $2 m+1$ powers of $f$.

4. The small and large period theorems. The commuting block maps problem was solved completely in [1] for block maps of the form $f\left(x_{0}, \ldots, x_{k}\right)=x_{0}+$ $\Pi_{i=1}^{k}\left(x_{i}+\delta_{i}\right)$ where $k \geqslant 2$. If the least period of the sequence of constants $\delta_{1} \ldots \delta_{k}$ is $p$ then $p \leqslant \frac{1}{2} k$ implies that $C^{*}(f)$ is the set of powers of $f$ while $p>\frac{1}{2} k$ implies $C^{*}(f)$ is the set of all sums of an odd number of powers of $f$. As a corollary to Theorem (3.5) the same result will now be proved to hold for block maps of the form $f=x_{0}+\prod_{i=1}^{k}\left(x_{m i}+\delta_{i}\right), k \geqslant 2, m \geqslant 1$.

Let $\mathscr{F}_{m n+1}^{*}$ denote the set of block maps $f\left(x_{0}, \ldots, x_{m n}\right) \in \mathscr{F}_{m n+1}$ which depend only on the variables $x_{j m}, 0 \leqslant j \leqslant n$. Strictly, $f \in \mathscr{F}_{m n+1}^{*}$ if and only if

$$
f\left(x_{0}, x_{1}, \ldots, x_{i-1}, 0, x_{i+1}, \ldots, x_{m n}\right)+f\left(x_{0}, x_{1}, \ldots, x_{i-1}, 1, x_{i+1}, \ldots, x_{m n}\right) \neq 0
$$


for some block $\left(x_{0}, x_{1}, \ldots, x_{i-1}, x_{i+1}, \ldots, x_{m n}\right)$ implies that $i=j m$ for some $j$. Thus if $f \in \mathscr{F}_{m n+1}^{*}$ and $\left(x_{0}, \ldots, x_{m n}\right),\left(y_{0}, \ldots, y_{m n}\right)$ are two blocks with $x_{j m}=y_{j m}$ for $0 \leqslant j \leqslant n$ then $f\left(x_{0}, \ldots, x_{m n}\right)=f\left(y_{0}, \ldots, y_{m n}\right)$. It follows that the map $\mathfrak{p}$ : $\mathscr{F}_{m n+1}^{*} \rightarrow \mathscr{F}_{n+1}$,

$$
\mathfrak{p} f\left(x_{0}, x_{m}, x_{2 m}, \ldots, x_{m n}\right)=f\left(x_{0}, x_{1}, x_{2}, \ldots, x_{n}\right)
$$

is well defined.

The map $\mathfrak{p}$ has the following properties.

(4.1) Lemma. If $f, g \in \mathscr{F}_{m n+1}^{*}$, then $f=g$ if and only if $\mathfrak{p} f=\mathfrak{p g}$. Also $\mathfrak{p}(f+g)=\mathfrak{p} f+\mathfrak{p} g$.

(4.2) Lemma. If $f \in \mathscr{F}_{m p+1}^{*}$ and $g \in \mathscr{F}_{m q+1}^{*}$, then $f \circ g \in \mathscr{F}_{m(p+q)+1}^{*}$ and $\mathfrak{p}(f \circ g)=\mathfrak{p} f \circ \mathfrak{p} g$.

Proof. Certainly $f \circ g \in \mathscr{F}_{m(p+q)+1}$. If $\phi\left(x_{1}, \ldots, x_{i_{\mu} m}\right)=x_{i_{1} m} x_{i_{2} m} \ldots x_{i_{\mu} m}$ then $\phi \circ g$ depends only on the variables $x_{\left(i_{\nu}+\lambda\right) m}, 0 \leqslant \lambda \leqslant q, 1 \leqslant \nu \leqslant \mu$. Since $R f$ is a sum of such functions $\phi$ and composition distributes on the left, $f \circ g$ depends only on the variables $x_{j m}, 0 \leqslant j \leqslant p+q$. Moreover, $\mathfrak{p}(\phi \circ g)=\mathfrak{p} \phi \circ \mathfrak{p} g$ and consequently $\mathfrak{p}(f \circ g)=\mathfrak{p} f \circ \mathfrak{p} g$.

(4.3) Lemma. Let $f=x_{0}+\prod_{i=1}^{k}\left(x_{i m}+\delta_{i}\right)=I+\prod_{i=1}^{k} T^{i m}\left(I+\delta_{i}\right), k \geqslant 2, m \geqslant 1$. If $g \in C^{*}(f)$ then $g \in \mathscr{F}_{m n+1}^{*}$ for some $n$. Moreover, $g \in C^{*}(f)$ if and only if $\mathfrak{p} g \in C^{*}(\mathfrak{p} f)$.

PROOF. The block map $R f$ is linear relative to $r_{1}, r_{2}, \ldots, r_{k-1}$ with $r_{i}=m$, $1 \leqslant i \leqslant k-1$, and $Q_{r_{k-1}} \ldots Q_{r_{2}} Q_{r_{1}} R f=T^{m} I+\delta_{i}$. Thus by Theorem (3.5), $g$ differs from a sum of an odd number of powers of $f$ by at most a constant. Hence, by Lemma (4.2), $g$ depends only on the variables $x_{j m}, 0 \leqslant j$. Then $g \circ f=f \circ g$ if and only if $\mathfrak{p}(g \circ f)=\mathfrak{p}(f \circ g)$ which occurs if and only if $\mathfrak{p} g \circ \mathfrak{p} f=\mathfrak{p} f \circ \mathfrak{p} g$.

(4.4) Theorem. Suppose $f=x_{0}+\prod_{i=1}^{k}\left(x_{m i}+\delta_{i}\right), k \geqslant 2, m \geqslant 1$, and suppose the least period of the sequence of constants $\delta_{1} \ldots \delta_{k}$ is $p$. Then $p \leqslant \frac{1}{2} k$ implies that $C^{*}(f)$ is the set of powers of $f$ while $p>\frac{1}{2} k$ implies that $C^{*}(f)$ is the set of all sums of an odd number of powers of $f$.

Proof. From Lemma (4.3) and Theorems [1] (5.3), [1] (6.11), if $p \leqslant \frac{1}{2} k$ and $g \in C^{*}(f)$ then $\mathfrak{p} g=(\mathfrak{p} f)^{n}=\mathfrak{p}\left(f^{n}\right)$ for some $n$. Thus $g=f^{n}$. Conversely, $f^{n} \in C^{*}(f)$. On the other hand, if $p>\frac{1}{2} k$ and $g \in C^{*}(f)$ then

$$
p g=\sum_{i=1}^{2 \lambda+1}(p f)^{n_{i}}=\mathfrak{p} \sum_{i=1}^{2 \lambda+1} f^{n_{i}}
$$

Thus $g=\sum_{i=1}^{2 \lambda+1} f^{n_{i}}$. Conversely, $\sum_{i=1}^{2 \lambda+1} f^{n_{i}} \in C^{*}(f)$.

\section{REFERENCES}

1. Ethan M. Coven, G. A. Hedlund and Frank Rhodes, The commuting block maps problem, Trans. Amer. Math. Soc. 249 (1979), 113-138.

2. G. A Hedlund, Endomorphisms and automorphisms of the shift dynamical system, Math. Systems Theory 3 (1969), 320-375. MR 41 \#4510.

Department of Mathematics, University of Southampton, Southampton S09 5NH, ENGland 